\title{
Theoretical Description for the Galvanostatic Electrode Pretreatment, Yielding Thiourea-Based Active Surface for Cathodic Conducting Polymer Deposition
}

\author{
Volodymyr V. Tkach ${ }^{1,2}$, Marta V. Kushnir ${ }^{1}$, Yana G. Ivanushko ${ }^{3}$, Anzhelika F. Molodianu ${ }^{3}$, Mariia P. \\ Mytchenok ${ }^{3}$, Oleksandra V. Ahafonova ${ }^{3}$, Sílvio C. De Oliveira ${ }^{2}$, Petro I. Yagodynets ${ }^{1}$, \\ Zholt O. Kormosh ${ }^{4}$, Yulia L. Bredikhina ${ }^{5,6}$, Olga V. Luganska ${ }^{7}$ \\ 1 Chernivtsi National University, 58000, Kotsyubyns'ky Str. 2, Chernivtsi, Ukraine \\ 2 Universidade Federal de Mato Grosso do Sul, Av. Sen. Felinto. Müller, 1555, C/P. 549, 79074-460, Campo Grande, MS, \\ Brazil \\ Bukovinian State Medical University, 58000, Teatral'na Sq. 9, Chernivtsi, Ukraine \\ 4 East Ukrainian National University, 43000, Voli Ave., 13, Lutsk, Ukraine \\ 5 Melitopol State Pedagogical University, 72300, Melitopol, Hetmanska Str., 20, Ukraine \\ 6 Khortytsya National Rehabilitation Academy, 69000, Naukove Mistechko Str., 59, Zaporizhzhia, Ukraine \\ 7 Zaporizhzhia National University, 69600, Zhukovsky Str., 66, Zaporizhzhia, Ukraine \\ * Correspondence: nightwatcher2401@ gmail.com; vvt2401@Chnu.edu.ua;
}

Scopus Author ID 55758299100

Received: 12.06.2020; Revised: 2.07.2020; Accepted: 3.07.2020; Published: 7.07.2020

\begin{abstract}
The system with the electrode modification by thiourea basic derivatives for conducting polymer indirect cathodic deposition has been described from the theoretical point of view. The electrode pretreatment process has been analyzed using the linear stability theory and bifurcation analysis. It was shown that the steady-state is realized rapidly and provides efficient surface pretreatment. On the other hand, the oscillatory and monotonic behavior, in this case, is also possible due to the double electric layer capacitance changes during the doping stage and electrochemical reaction.
\end{abstract}

Keywords: conducting polymers; indirect cathodic electropolymerization; surface pretreatment; thiourea; stable steady-state.

(C) 2020 by the authors. This article is an open-access article distributed under the terms and conditions of the Creative Commons Attribution (CC BY) license (https://creativecommons.org/licenses/by/4.0/).

\section{Introduction}

Electropolymerization is one of the most used conducting polymer synthesis techniques [1-6]. The electrosynthetized polymers have some advantages while compared to the chemically synthesized polymers, like enhanced conductivity, flexibility, and versatility in modification for specific purposes and high sensing abilities. Depending on the participation of the monomer in the electrochemical stage, the electropolymerization may be direct or indirect.

The indirect electropolymerization, in which the electrochemical stage does not involve the proper monomer, but yields an active substance, promoting the chain formation and propagation, sometimes maybe more flexible than the direct one [5 - 6]. Independently on the monomer nature, it may be realized either cathodically or anodically. Moreover, it permits a one-stage realization of the processes like metal-polymer co-deposition, usually realized in two stages [7-10]. 
Nevertheless, in some cases the electropolymerization, be it anodic or cathodic, may require the electrode pretreatment [11-14], which may include not only physical or physicochemical processes like adsorption, chemical or electrochemical polish, but also be a complex process involving chemical and electrochemical stages (material doping, enzyme entrapment, graphene electrooxidation with further reduction, sulfide passivation). The aim of the electrode pretreatment is to enhance the polymer stability, adhesion, to modify by the proper way its behavior. If the electrosynthesis is indirect, the pretreatment may also be used to stabilize the initiating species.

For example, thiourea and analogs, used to prevent the hydrogen evolution in cathodic processes and to augment the polymer adhesion, may be electrochemically reduced, yielding amino-mercaptans or elementary sulfur and diamines. Afterward, the resulting compounds may be doped by the initiating ion, preparing the surface for the electropolymerization process. These processes may be accompanied by the electrochemical instabilities (oscillatory and monotonic) [15 - 18], capable of causing drastic changes in the resulting polymer changes.

In this work, the theoretical description of the system described above is exposed. Through mathematical modeling and analysis, it is possible to investigate the steady-state stability conditions, like also to foresee the occurrence of electrochemical instabilities affecting the system. Also, the behavior of this system will be compared to similar ones [19-21].

\section{Materials and Methods}

\subsection{System and its modeling.}

The surface pretreatment is realized in acidic media. By this, the thiourea is reduced to form the sulfur and methylene diamine, according to the reaction (1):

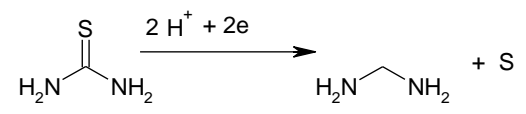

Methylenediamine is furtherly protonated in the presence of the anion, yielding a salt (2):

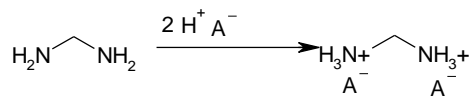

By this, the salt is formed, the conductivity is enhanced, and the hydrogen evolution is impeded.

Methylenediamine is used as a host matrix, yet doped by an ion, furtherly used as an initiator of the indirect electropolymerization process or even chemically assisted deposition. Thiourea may be easily substituted by thio- and semithiosquaraine dyes with basic nitrogen, mercaptoanilines, thiosemicarbazones, aminothiophenes, mercaptoazaarenes, and analogous compounds.

Due to the exposed, supposing the relative excess of the protons, the behavior of the system in galvanostatic mode will be described by three variables:

$\mathrm{c}$ - the initiating ion concentration in the pre-surface layer;

$\mathrm{m}$ - the undoped methylenediamine surface coverage degree

$\mathrm{q}$ - the anode charge density.

To simplify the modeling, we suppose that the reactor is intensively stirred, so we can neglect the convection flow. Also, we assume that the background electrolyte is in excess, so we can neglect the migration flow. The diffusion layer is supposed to be of a constant thickness, equal to $\delta$, and the concentration profile in it is supposed to be linear. 
The bivariant equation-set, describing the system will be exposed as:

$$
\left\{\begin{array}{c}
\frac{d c}{d t}=\frac{2}{\delta}\left(\frac{\Delta}{\delta}\left(c_{0}-c\right)-r_{d}\right) \\
\frac{d m}{d t}=\frac{1}{M}\left(r_{r}-r_{d}\right) \\
\frac{d t}{d t}=i-i_{F}
\end{array}\right.
$$

where $\Delta$ is diffusion coefficient, $\mathrm{c}_{0}$ is the initiator bulk concentration in the pre-surface layer, $\mathrm{i}_{\mathrm{F}}$ is Faradaic current, $\mathrm{M}$ is the methylenediamine maximal surface concentration, and the parameters $r$ correspond to the reduction and doping rate, capable of being described as:

$$
\begin{gathered}
r_{r}=k_{r}(1-m) \exp \left(-\frac{2 F \varphi_{0}}{R T}\right) \\
r_{d}=k_{d} m c^{2} \exp (-\alpha m) \\
i_{F}=2 F k_{r}(1-m) \exp \left(-\frac{2 F \varphi_{0}}{R T}\right)
\end{gathered}
$$

In which the parameters $\mathrm{k}$ are the correspondent rate constants, $\varphi_{0}$ is the potential slope in the double electric layer (DEL), $\alpha$ is the parameter, relating its capacitance to the doping degree, $\mathrm{F}$ is the Faraday number, $\mathrm{R}$ is the universal gas constant, and $\mathrm{T}$ is the absolute temperature.

As a matter of fact, this system will be similar to the systems with the electrochemical determination by doping and with the surface-assisted indirect electropolymerization [20 - 21]. Nevertheless, as we have chemical doping instead of electrochemical (like it was during the direct electropolymerization of a monomer in the presence of the initiating ion), the DEL influences of the process will be slightly different, as shown below.

\section{Results and Discussion}

In order to investigate the system with the electropolymerization surface pretreatment by thiourea reduction and doping of its product, we analyze the equation-set (3) by means of the linear stability theory. The steady-state Jacobian matrix members for this system may be exposed as:

in which:

$$
\left(\begin{array}{lll}
a_{11} & a_{12} & a_{13} \\
a_{21} & a_{22} & a_{23} \\
a_{31} & a_{32} & a_{33}
\end{array}\right)
$$

$$
\begin{gathered}
a_{11}=\frac{2}{\delta}\left(-\frac{\Delta}{\delta}-2 k_{d} m c \exp (-\alpha m)\right) \\
a_{12}=\frac{2}{\delta}\left(-k_{d} c^{2} \exp (-\alpha m)+k_{d} m c^{2} \exp (-\alpha m)\right) \\
a_{13}=0 \\
a_{21}=\frac{1}{M}\left(-2 k_{d} m c \exp (-\alpha m)\right) \\
a_{22}=\frac{1}{M}\left(-k_{r} \exp \left(-\frac{2 F \varphi_{0}}{R T}\right)-j k_{r}(1-m) \exp \left(-\frac{2 F \varphi_{0}}{R T}\right)-k_{d} c^{2} \exp (-\alpha m)+\right. \\
\left.\alpha k_{d} m c^{2} \exp (-\alpha m)\right) \\
a_{23}=\frac{1}{M}\left(-l k_{r}(1-m) \exp \left(-\frac{2 F \varphi_{0}}{R T}\right)\right) \\
a_{32}=-2 F\left(k_{r} \exp \left(-\frac{2 F \varphi_{0}}{R T}\right)+j k_{r}(1-m) \exp \left(-\frac{2 F \varphi_{0}}{R T}\right)\right) \\
a_{32}=2 F\left(-k_{r} \exp \left(-\frac{2 F \varphi_{0}}{R T}\right)-j k_{r}(1-m) \exp \left(-\frac{2 F \varphi_{0}}{R T}\right)\right)
\end{gathered}
$$




$$
a_{33}=-2 F\left(l k_{r}(1-m) \exp \left(-\frac{2 F \varphi_{0}}{R T}\right)\right)
$$

Observing the elements (8), (13) and (17), we may conclude that the oscillatory behavior in this system is possible. Moreover, its probability is enhanced in relation to the cathodic indirect solution-initiated electropolymerization [19], but is equal to that of the systems with the surface-initiated polymerization (to which the surface is hereby prepared) [2021]. In galvanostatic mode, there are three elements capable of being positive, against two for potentiostatic mode. As in [20-21], the elements $j k_{r}(1-m) \exp \left(-\frac{2 F \varphi_{0}}{R T}\right)>0$ if $\mathrm{j}<0$, related to the DEL influences on the electrochemical stage, and $\alpha k_{d} m c^{2} \exp (-\alpha m)>0$, if $\alpha>0$, related to the DEL capacitance impact of the chemical doping, is responsible for oscillatory behavior. Another factor is described by the positivity of $-2 F\left(l k_{r}(1-m) \exp \left(-\frac{2 F \varphi_{0}}{R T}\right)\right)>$ 0 if $1<0$. All of the mentioned factors act either during the assisted electropolymerization or during the surface pretreatment.

In order to investigate the steady-state stability, we apply the Routh-Hurwitz criterion to the equation-set (3). Avoiding the cumbersome expressions, we introduce new variables and, therefore, describe the Jacobian matrix determinant as:

$$
\frac{4 F}{\delta M}\left|\begin{array}{ccc}
-\kappa-\mu & -v & 0 \\
-\mu & -v-\xi & -\lambda \\
0 & -\xi & -\lambda
\end{array}\right|
$$

Opening the brackets, we apply the Det $\mathrm{J}<0$ requisite, salient from the criterion and, resolving the inequation relatively to the electrochemical parameter $\lambda$, obtain the steady-state stability condition, expressed as (19):

$$
-\lambda(\kappa \nu+2 \kappa \xi+\mu \xi-v \xi)<0
$$

This inequation depicts either diffusion or kinetically controlled electrosynthetical system. It is warranted to be satisfied in the case of the fragility of the DEL influences of chemical and electrochemical stages. Really, the positive values of $\lambda, v$, and $\xi$ lead the left part of the inequation to more negative values, stabilizing the steady-state and leading to the appearance of a developed catalytic surface with "cabbage-like" morphology. It will provide the deposition of a similar polymer structure, as observed in [20-21].

The monotonic instability for this system is possible. It is defining the margin between stable steady-states and unstable states, and its conditions of appearance will be depicted as (20):

$$
-\lambda(\kappa \nu+2 \kappa \xi+\mu \xi-v \xi)=0
$$

While prepared, the pretreated surface may assist either the indirect electropolymerization or even chemically induced surface polymerization, depending on the monomer, synthesis conditions, and the dopant. While used in the indirect electropolymerization, the process will be described by the models, exposed in [20-21].

\section{Conclusions}

From the theoretical investigation of the assisted electropolymerization electrode pretreatment by thiourea reduction in the presence of an initiating dopant ion, it is possible to conclude that:

The electrode pretreatment is a diffusion-controlled process, in which the steady-state mode is easy to obtain and maintain. 
The electrode pretreatment yields an active surface capable of supporting efficiently either electrochemical or chemical polymerization, augmenting the conductivity and adhesion of the resulting polymer.

The oscillatory behavior in this system is possible. As in the case of the surface-assisted indirect electropolymerization, it will be caused by double electric layer influences of both electrochemical stage and chemical doping reaction.

\section{Funding}

This research received no external funding.

\section{Acknowledgments}

This research has no acknowledgment.

\section{Conflicts of Interest}

The authors declare no conflict of interest.

\section{References}

1. Angeli, A.; Alessandri, L.J.G.C.I. The electrochemistry of conducting polymers. 1916, 46, 279-285.

2. Dall'Olio, A.; Dascola, G.; Vacara, V.; Bocchi, V.J.C.A.S.P. Resonance paramagnetique electronique et conductivité d'un noir d'oxypyrrol electrolytique. 1968, 267, 433-435.

3. Sadki, S.; Schottland, P.; Brodie, N.; Sabouraud, G.J.C.S.R. The mechanisms of pyrrole electropolymerization. 2000, 29, 283-293, https://doi.org/10.1039/A807124A.

4. Ağın, F.J.A.L. Electrochemical determination of amoxicillin on a poly (acridine orange) modified glassy carbon electrode. 2016, 49, 1366-1378, https://doi.org/10.1080/00032719.2015.1101602.

5. Jung, Y.; Singh, N.; Choi, K.S. thodic Deposition of Polypyrrole Enabling the One-Step Assembly of MetalPolymer Hybrid Electrodes. Angew. Chem. Int. Ed. 2009, 121, 8481-8484, https://doi.org/10.1002/ange.200903596.

6. German, N.; Popov, A.; Ramanaviciene, A.; Ramanavicius, A.J.N. Enzymatic Formation of Polyaniline, Polypyrrole, and Polythiophene Nanoparticles with Embedded Glucose Oxidase. 2019, 9, https://doi.org/10.3390/nano9050806.

7. Liu, Z.; Liang, F.; Zhang, N.; Liu, Y.J.I.J.E.S. Preparation of Manganese Dioxide/Polypyrrole Composite by W/O Miniemulsion and Its Electrochemical Performance. J. Electrochem. Sci. 2018, 13, 6584-6597, https://doi.org/10.1039/C4NR06978A.

8. Wang, H.; Ma, N.; Yan, Z.; Deng, L.; He, J.; Hou, Y.; Jiang, Y.; Yu, G.J.N. Cobalt/polypyrrole nanocomposites with controllable electromagnetic properties. Nanoscale 2015, 7, 7189-7196, https://doi.org/10.1039/C4NR06978A.

9. Hakimi, N.; Zouaoui, A.; Satour, F.Z.; Sahari, A.; Zegadi, A. Electrochemical Synthesis and Properties of the Composite Material ITO/Polypyrrole-Benzoic: Cobalt for Electronic Storage Applications. Journal of Inorganic and Organometallic Polymers and Materials 2020, 30, 330-336, https://doi.org/10.1007/s10904019-01191-3.

10. Ananda, S.R.; Murugendrappa, M.J.I.J.o.M.S. Thermal Studies of Polypyrrole and Cobalt Aluminum Oxide Nano Composites. Int. J. Mat. Sci., 2017, 12, 247.

11. Bazzaoui, M.; Martins, J.; Bazzaoui, E.; Reis, T.; Martins, L.J.J.o.a.e. Pyrrole electropolymerization on copper and brass in a single-step process from aqueous solution. 2004, 34, 815-822, https://doi.org/10.1023/B:JACH.0000035610.10869.43.

12. Tsamouras, D.; Dalas, E.; Sakkopoulos, S.; Vitoratos, E.J.A.s.s. Characterization of sulfide/polyaniline and sulfide/polypyrrole contacts. 1993, 65, 388-393, https://doi.org/10.1016/0169-4332(93)90691-4.

13. Hamilton, A. The formation and Characterization of a Polypyrrole Based Sensor for the Detection of Urea, Ph.D. Thesis, National University of Ireland, Dublin, 2012.

14. Dalmoro, V.; Cedron, S.; Azambuja, K.S.; Castagno, K.R.L. Polypyrrole Film Doped with Corrosion Inhibitors, Electropolymerized on AA 1100 Mat. Res. 2009, 22. e20180919

15. Das, I.; Goel, N.; Agrawal, N.R.; Gupta, S.K.J.T.J.o.P.C.B. Growth patterns of dendrimers and electric potential oscillations during electropolymerization of pyrrole using mono-and mixed surfactants. 2010, 114, 12888-12896, https://doi.org/10.1021/jp105183q. 
16. Bazzaoui, M.; Bazzaoui, E.; Martins, L.; Martins, J.J.S.m. Electropolymerization of pyrrole on zinc-leadsilver alloys electrodes in acidic and neutral organic media. Synth. Met. 2002, 130, 73-83.

17. Das, I.; Goel, N.; Gupta, S.K.; Agrawal, N.R.J.J.o.E.C. Electropolymerization of pyrrole: Dendrimers, nanosized patterns and oscillations in potential in presence of aromatic and aliphatic surfactants. J. Electroanal. Chem. 2012, 670, 1-10.

18. Ba-Shammakh, M.S. Thes. Electropolymerization of pyrrole on mild steel for corrosion protection. Ph. D. King Fahd University of Petroleum and Minerals, Dharan, Saudi Arabia, 2002.

19. Tkach, V.V.; Storoshchuk, N.M.; Romaniv, L.V.; De Oliveira, S.C.; Luganska, O.V.; Gala, H.B.; Tchikuala, E.F.; Yagodynets, P.I.Vietnam.J,. Chem.. The theoretical evaluation of the poly (3, 4'dimetylpyrrole) cathodic electrodeposition assisted by manganate ions. 2018, 56, 440-444, https://doi.org/10.1002/vjch.201800027.

20. Tkach, V.V.; Storoshchuk, N.M.; de Oliveira, S.C. et al. The Theoretical Evaluation of the Poly $(3,4-$ dimethylpyrrole) Cathodic Electrodeposition, Assisted by Manganate Ions, Intercalated into a Conducting Polymer. Appl. J. Env. Eng. Sci. 2018, 4, 328.

21. Tkach, V.V.; Kushnir, M.V.; Storoshchuk, N.M. et al.The Theoretical Description of Cathodic Pyrrole Electrodeposition, Assisted by a Dichromate of a Novel Triazolic Derivative, Rhazes 2018, 2, 33 